Proceedings of the Institute of Mathematics and Mechanics,

National Academy of Sciences of Azerbaijan

Volume 46, Number 1, 2020, Pages 115-128

https://doi.org/10.29228/proc. 22

\title{
ON DUALITY IN OPTIMAL CONTROL PROBLEMS WITH SECOND-ORDER DIFFERENTIAL INCLUSIONS AND INITIAL-POINT CONSTRAINTS
}

\author{
ELIMHAN N. MAHMUDOV AND MISIR J. MARDANOV
}

\begin{abstract}
The paper deals with the optimal control problem described by second-order differential inclusions. Based on the infimal convolution concept of convex functions, dual problems for differential inclusions are constructed and the results of duality are proved. In this case, it turns out that Euler-Lagrange type inclusions are "duality relations" for both primary and dual problems. In particular, the linear second-order optimal control problem with the Mayer functional is considered. This problem shows that maximization in the dual problems is realized over the set of solutions of the adjoint equation. Finally, we construct the dual problem to the problem with the second-order polyhedral differential inclusion.
\end{abstract}

\section{Introduction}

Many extremal problems, for example, classical problems of optimal control, differential games, models of economic dynamics, macroeconomic problems, etc. are described in terms of set-valued mappings and form a component part of the modern mathematical theory of controlled dynamical systems and mathematical economics $[3,8,17,18]$. The first and second-order ordinary and partial differential inclusions, naturally arising from certain physical and control problems, have attracted the attention of many researchers, and as a result, various qualitative problems, including the existence results have been considered by many authors (see [1, 5, 6], [9]-[11], [15, 21, 22] and references therein). The paper [12] studies sufficient conditions of optimality for the Cauchy problem of fourth-order differential inclusions. Mainly our purpose is to derive sufficient optimality conditions for mentioned problems with fourth-order differential inclusions (DFIs) and transversality conditions. The results reveal that the proposed method is very accurate and efficient. The paper [21] concerns optimal control of discontinuous differential inclusions of the normal cone type governed by a generalized version of the Moreau sweeping process with control functions acting in both nonconvex moving sets and additive perturbations.

In the papers [11]-[16], for optimal control problems of higher order discrete processes and DFIs with the use of locally adjoint mappings (LAMs) the necessary

2010 Mathematics Subject Classification. 34A60, 49N15, 49M25, 90C46.

Key words and phrases. Duality, conjugate, Euler-Lagrange, polyhedral, sufficient conditions. 
and sufficient conditions of optimality are formulated. Along with these the duality theory plays a fundamental role in the analysis of optimization and variational problems. The reader can consult $[2,3,20]$ and their references for more details on this topic. It not only provides a powerful theoretical tool in the analysis of these problems, but also paves the way to designing new algorithms for solving them. A key player in any duality framework is the Legendre-Fenchel conjugate transform. Often, duality is associated with convex problems, yet it turns out that duality theory also has a fundamental impact even on the analysis of nonconvex problems. The work [10] is devoted to optimization of so-called first-order partial DFIs in the gradient form on a square domain. In the Euler-Lagrange form, necessary and sufficient conditions are derived for the discrete-approximate and partial DFIs, respectively. The duality theorems are proved and duality relation is established. In the present work, the optimality conditions for a second-order DFIs together with their duality approach were considered for the first time. To the best of our knowledge, there are a few papers (see $[9,10,20]$ and the references therein) devoted to duality problems of first order DFIs. Building on these results, we then treat dual results according to the dual operations of addition and infimal convolution of convex functions $[2,7,8]$.

Thus, the present paper is dedicated to one of the difficult and interesting fields construction of duality of optimization problems with second-order ordinary discrete and DFIs. The posed problems and their dualities are new. The paper is organized in the following order:

In Section 2, the needed facts and supplementary results from the book of Mahmudov[8] are given; Hamiltonian function $H$ and argmaximum sets of a setvalued mapping $F$, the LAM, infimal convolution of proper convex functions, conjugate function for Hamiltonian function taken with a minus sign are introduced and the problems for second-order DFIs $\left(P_{C}\right)$ with initial point constraints are formulated.

In Section 3 necessary and sufficient conditions of optimality for second-order DFIs are formulated, the dual problem for second-order DFIs $\left(P_{C}\right)$ is constructed. In what follows, we prove that if $\alpha$ and $\alpha^{*}$ are the values of primary and dual problems, respectively, then $\alpha \geq \alpha^{*}$ for all feasible solutions. Moreover, if a certain "nondegeneracy condition", that is standard condition of convex analysis on existence of interior point, is satisfied, then the existence of a solution to one of these problems implies the existence of a solution to the other problem, where $\alpha=\alpha^{*}$, and in the case where $\alpha>\infty$ the dual problem has a solution. Finally, duality relationship between a pair of optimization problems with initial point constraint established; it is proved that the Euler-Lagrange type adjoint inclusion at the same time is a dual relation. Aft the of this section we consider a Mayer problem with second-order linear optimal control problem. The considered problem shows that maximization in the dual problems is realized over the set of solutions of the adjoint equation. In addition, the optimal values in the primary convex $\left(P_{C}\right)$ and the dual concave $\left(P_{C}^{*}\right)$ problems are equal: $\inf \left(P_{C}\right)=\sup \left(P_{C}^{*}\right)$. Thus, it is proved that the Euler-Lagrange type adjoint inclusion at the same time is a dual relation, that is a pair of solutions of primary and dual problems satisfies this relation. 
In Section 4 we establish the dual problem $\left(P L^{*}\right)$ to the problem with the following second-order polyhedral differential inclusion $F(x, y)=\{z: A x+B y-$ $C z \leq d\}$, where $A, B, C$ and $d$ are matrices of appropriate dimensions. Here is proved that $M_{F}\left(x^{*}, y^{*}, z^{*}\right)=-\langle d, \lambda\rangle$ and the dual problem $\left(P L^{*}\right)$ is expressed by transposed matrices $A^{*}, B^{*}, C^{*}$.

\section{Needed Facts and Problem Statement}

Further, for the convenience of the reader, all the necessary concepts, definitions of a convex analysis can be found in the book of Mahmudov [8]. Let $\mathbb{R}^{n}$ be a $n$-dimensional Euclidean space, $\langle x, u\rangle$ be an inner product of elements $x, y \in \mathbb{R}^{n}$ and $(x, y)$ be a pair of $x, y$. Assume that $G: \mathbb{R}^{n} \times \mathbb{R}^{n} \rightrightarrows \mathbb{R}^{n}$ is a set-valued mapping from $\mathbb{R}^{2 n}=\mathbb{R}^{n} \times \mathbb{R}^{n}$ into the set of subsets of $\mathbb{R}^{n}$. Then $G: \mathbb{R}^{2 n} \rightrightarrows \mathbb{R}^{n}$ is convex if its $g p h G=\{(x, y, z): z \in G(x, y)\}$ is a convex subset of $\mathbb{R}^{3 n}$. The set-valued mapping $G$ is convex closed if its graph is a convex closed set in $\mathbb{R}^{3 n}$. The domain of $G$ is denoted by $\operatorname{dom} G$ and is defined as follows $\operatorname{dom} G=\{(x, y): G(x, y) \neq \varnothing\}$. $G$ is convex-valued if $G(x, y)$ is a convex set for each $(x, y) \in \operatorname{dom} G$.

Let us introduce the Hamiltonian function and argmaximum set for a setvalued mapping $G$

$$
\begin{array}{r}
H_{G}\left(x, y, z^{*}\right)=\sup _{z}\left\{\left\langle z, z^{*}\right\rangle: z \in G(x, y)\right\}, z^{*} \in \mathbb{R}^{n}, \\
G_{A}\left(x, y ; z^{*}\right)=\left\{z \in G(x, y):\left\langle z, z^{*}\right\rangle=H_{G}\left(x, y, z^{*}\right)\right\},
\end{array}
$$

respectively. For convex $G$ we set $H_{G}\left(x, y, z^{*}\right)=-\infty$ if $G(x, y)=\varnothing$. As usual, $W_{A}\left(x^{*}\right)$ is a support function of the set $A \subset \mathbb{R}^{n}$, i.e.,

$$
W_{A}\left(x^{*}\right)=\sup _{x}\left\{\left\langle x, x^{*}\right\rangle: x \in A\right\}, x^{*} \in \mathbb{R}^{n} .
$$

Let int $A$ be the interior of the set $A \subset \mathbb{R}^{3 n}$ and $r i A$ be the relative interior of the $\operatorname{set} A$ i.e. the set of interior points of $A$ with respect to its affine hull $A f f A$.

The convex cone $K_{A}\left(w_{0}\right), w_{0}=\left(x_{0}, y_{0}, z_{0}\right)$, is called the cone of tangent directions at a point $w_{0} \in A$ to the $\operatorname{set} A$ if from $\bar{w}=(\bar{x}, \bar{y}, \bar{z}) \in K_{A}\left(w_{0}\right)$ it follows that is a tangent vector to the set $A$ at point $w_{0} \in A$, i.e., there exists such function $\eta: \mathbb{R}^{1} \rightarrow \mathbb{R}^{3 n}$ that $w_{0}+\lambda \bar{w}+\eta(\lambda) \in A$ for sufficiently small $\lambda>0$ and $\lambda^{-1} \eta(\lambda) \rightarrow 0$, as $\lambda \downarrow 0$.

A function $\varphi$ is called a proper function if it does not assume the value $-\infty$ and is not identically equal to $+\infty$. Obviously, $\varphi$ is proper if and only if $d o m \varphi \neq \varnothing$ and $\varphi(x, y)$ is finite for $(x, y) \in \operatorname{dom} \varphi=\{(x, y): \varphi(x, y)<+\infty$.

In general, for a set-valued mapping $G$ a set-valued mapping $G^{*}(\cdot, x, y, z)$ : $\mathbb{R}^{n} \rightrightarrows \mathbb{R}^{2 n}$ defined by

$$
G^{*}\left(z^{*} ;(x, y, z)\right):=\left\{\left(x^{*}, y^{*}\right):\left(x^{*}, y^{*},-z^{*}\right) \in K_{g p h G}^{*}(x, y, z)\right\},
$$

is called the LAM to a set-valued $G$ at a point $(x, y, z)$, where $K_{g p h G}^{*}(x, y, z)$ is the dual to the cone of tangent directions $K_{g p h G}(x, y, z)$. We provide another definition of LAM to mapping $G$ which is more relevant for further development 


$$
\begin{aligned}
& G^{*}\left(z^{*} ;(x, y, z)\right):=\left\{\left(x^{*}, y^{*}\right): H_{G}\left(x_{1}, y_{1}, z^{*}\right)-H_{G}\left(x, y, z^{*}\right) \leq\left\langle x^{*}, x_{1}-x\right\rangle\right. \\
& \left.\quad+\left\langle y^{*}, y_{1}-y\right\rangle, \forall\left(x_{1}, y_{1}\right) \in \mathbb{R}^{2 n}\right\},(x, y, z) \in g p h G, z \in G_{A}\left(x, y, z^{*}\right) .
\end{aligned}
$$

We note that the coderivative concept of Mordukhovich [19] is essentially different for nonconvex mappings. The notion of coderivative has been introduced for set-valued mappings in terms of the basic normal cone to their graphs. In the most interesting settings for the theory and applications, coderivatives are nonconvex-valued and hence are not tangentially /derivatively generated. This is the case of the first coderivative for general finite dimensional set-valued mappings for the purpose of applications to optimal control. The main advantage of the definition of LAM is its simplicity. Clearly, for the convex mapping the Hamiltonian $H\left(\cdot, \cdot, z^{*}\right)$ is concave and the latter and previous definitions of LAMs coincide. However, for the smooth and convex maps the two notions are equivalent.

Definition 2.1. A function $\varphi(x, y)$ is said to be closed, if its epigraph epi $\varphi=$ $\left\{\left(x^{0}, x, y\right): x^{0} \geq \varphi(x, y)\right.$ is a closed set.

Definition 2.2. The function $\varphi^{*}\left(x^{*}, y^{*}\right)=\sup _{x, y}\left\{\left\langle x, x^{*}\right\rangle+\left\langle y, y^{*}\right\rangle-\varphi(x, y)\right\}$ is called the conjugate of $\varphi$. It is clear to see that the conjugate function is closed and convex.

Let us denote

$$
M_{G}\left(x^{*}, y^{*} z^{*}\right)=\inf _{x, y, z}\left\{\left\langle x, x^{*}\right\rangle+\left\langle y, y^{*}\right\rangle-\left\langle z, z^{*}\right\rangle:(x, y, z) \in g p h G\right\},
$$

that is, for every $(x, y) \in \mathbb{R}^{2 n}$

$$
M_{G}\left(x^{*}, y^{*} z^{*}\right) \leq\left\langle x, x^{*}\right\rangle+\left\langle y, y^{*}\right\rangle-H_{G}\left(x, y, z^{*}\right) .
$$

It is clear that the function

$$
M_{G}\left(x^{*}, y^{*} z^{*}\right)=\inf _{x, y}\left\{\left\langle x, x^{*}\right\rangle+\left\langle y, y^{*}\right\rangle-H_{G}\left(x, y, z^{*}\right)\right\}
$$

is a support function taken with a minus sign. Besides, it follows that for a fixed $z^{*}$

$$
M_{G}\left(x^{*}, y^{*} z^{*}\right)=-\left[H_{G}\left(\cdot, \cdot, z^{*}\right)\right]^{*}\left(x^{*}, y^{*}\right)
$$

that is, $M_{G}$ is the conjugate function for $H_{G}\left(\cdot, \cdot, z^{*}\right)$ taken with a minus sign.

Definition 2.3. We recall that the operation of infimal convolution $\oplus$ of functions $f_{1}, f_{2}$ is defined as follows

$$
\left(f_{1} \oplus f_{2}\right)(u)=\inf \left\{f_{1}\left(u^{1}\right)+f_{2}\left(u^{2}\right): u^{1}+u^{2}=u\right\}, u^{i} \in \mathbb{R}^{n}, i=1,2 .
$$

The infimal convolution $\left(f_{1} \oplus f_{2}\right)$ is said to be exact provided the infimum above is attained for every $u \in \mathbb{R}^{n}$. One has $\left.\operatorname{dom}\left(f_{1} \oplus f_{2}\right)=\operatorname{dom} f_{1}+\operatorname{dom} f_{2}\right)$. Besides for a proper convex closed functions $f_{1}, f_{2}$ their infimal convolution $\left(f_{1} \oplus f_{2}\right)$ is convex and closed (but not necessarily proper). If $f_{1}, f_{2}$ are functions not 
identically equal to $+\infty$, then $\left(f_{1} \oplus f_{2}\right)^{*}=f_{1}^{*}+f_{2}^{*}$. Thus, the conjugate of infimal convolution is the sum of the conjugates and this holds without any requirement on the convex functions. The operations + and $\oplus$ are thus dual to each other with respect to taking conjugates.

In Section 3 we deal with the Mayer problem for $\left(P_{C}\right)$ type of the evolution DFIs:

$$
\begin{aligned}
\text { infimum } & \varphi\left(x(1), x^{\prime}(1)\right), \\
\left(P_{C}\right) \quad & x^{\prime \prime}(t) \in F\left(x(t), x^{\prime}(t), t\right), \text { a.e. } t \in[0,1], \\
& x(0) \in Q_{0}, x^{\prime}(0) \in Q_{1} .
\end{aligned}
$$

Here $F(\cdot, t): \mathbb{R}^{n} \rightrightarrows \mathbb{R}^{n}$ is a time dependent set-valued mapping, $\varphi$ is continuous $\varphi: \mathbb{R}^{2 n} \rightarrow \mathbb{R}^{1}, Q_{i} \subseteq \mathbb{R}^{n}(i=0,1)$ are nonempty subsets. The problem is to find an arc $\tilde{x}(\cdot)$ of the problem (2.1)-(2.3) satisfying (2.2) almost everywhere (a.e.) on $[0,1]$ and the initial-point constraints $(2.3)$ on $[0,1]$ that minimizes the Mayer functional $\varphi\left(x(1), x^{\prime}(1)\right)$. We label this problem as $\left(P_{C}\right)$. Here, a feasible trajectory $x(\cdot)$ is understood to be an absolutely continuous function on a time interval $[0,1]$ together with the first order derivatives for which $x^{\prime \prime}(\cdot) \in L_{1}^{n}([0,1])$. Obviously, such class of functions is a Banach space, endowed with the different equivalent norms. For example, $\|x(\cdot)\|=\sum_{k=0}^{1}\left|x^{(k)}(0)\right|+\left\|x^{\prime \prime}(\cdot)\right\|_{1}$ or $\left\|x^{\prime \prime}(\cdot)\right\|=$ $\sum_{k=0}^{2}\left\|x^{(k)}(0)\right\|_{1}$, where $\left\|x^{(k)}(0)\right\|_{1}=\int_{0}^{1}\left|x^{(k)}(t)\right| d t$ and $|x|$ is an Euclidean norm in $\mathbb{R}^{n}$.

Note that an absolutely continuous function is a.e. differentiable function and in the Lebesgue sense, its derivative function is integrable. But not always a function having a.e. integrable derivative is absolutely continuous. For example, the Cantor function [4] is the such a function; it is well known that the Cantor function $f:[0,1] \rightarrow[0,1]$ is a.e. differentiable, where $f^{\prime}(t)=0, f(0)=0, f(1)=$ 1. Then the condition of the familiar Lebesgue theorem [4] $\int_{0}^{1} f^{\prime}(t) d t=f(1)-f(0)$ is not satisfied.

\section{The Dual Problem for Convex DFIs}

Here we treat dual results according to the dual operations of addition and infimal convolution of convex functions $[7,8]$. But the construction of the duality problem would lead us too far astray from the main themes of this paper and is therefore omitted. And in this sense the obtained results here are only the visible part of the "icebergs". In order to establish a dual problem to the main problem $\left(P_{C}\right)$, we have used a limiting process in dual problem for a discrete-approximate problem to continuous problem $\left(P_{C}\right)$; by passing to the formal limit as a discrete step tends to zero, the obtained maximization problem will be the dual problem to the previous continuous convex problem $\left(P_{C}\right)$ :

$$
\begin{aligned}
& \sup _{x^{*}(\cdot), v^{*}(\cdot)}\left\{-\varphi^{*}\left(v^{*}(1)+x^{*^{\prime}}(1),-x^{*}(1)\right)+\int_{0}^{1}\left[M _ { F ( \cdot , t ) } \left(x^{*^{\prime \prime}}(t)\right.\right.\right. \\
& \left.\left.\left.+v^{*^{\prime}}(t), v^{*}(t), x^{*}(t)\right)\right] d t-W_{Q_{0}}\left(-v^{*}(0)-x^{*^{\prime}}(0)\right)-W_{Q_{1}}\left(x^{*}(0)\right)\right\} .
\end{aligned}
$$


Furthermore, we assume that $x^{*}(t), t \in[0,1]$ is absolutely continuous function together with the first order derivatives and $x^{*^{\prime \prime}}(\cdot) \in L_{1}^{n}([0,1])$. Moreover, $v^{*}(\cdot)$ is absolutely continuous and $v^{*^{\prime}}(\cdot) \in L_{1}^{n}([0,1])$.

In order that to prove the duality theorem we need to formulate the duality relation. We shall prove that the duality relation is an adjoint inclusion of Euler-Lagrange type. To this end, in the following theorem, sufficient optimality conditions are formulated for convex second-order DFIs with convex nonfunctional initial point constraints $\left(P_{C}\right)$. These conditions are more precise since they involve useful forms of the Weierstrass-Pontryagin condition and secondorder Euler-Lagrange type adjoint inclusions. In the reviewed results this effort culminates in Theorem 3.1.

First, we formulate the reminded second-order Euler-Lagrange type adjoint inclusion and transversality conditions for the problem $\left(P_{C}\right)$

(a) $\left(x^{*^{\prime \prime}}(t)+v^{*^{\prime}}(t), v^{*}(t)\right) \in F^{*}\left(x^{*}(t) ;\left(\tilde{x}(t), \tilde{x}^{\prime}(t), \tilde{x}^{\prime \prime}(t)\right), t\right)$, a.e. $t \in[0,1]$,

where

(b) $\quad \tilde{x}^{\prime \prime}(t) \in F_{A}\left(\tilde{x}(t), \tilde{x}^{\prime}(t) ; x^{*}(t), t\right)$, a.e. $t \in[0,1]$.

The transversality conditions at the endpoints $t=0$ and $t=1$ consist of the following

$$
\begin{gathered}
(c) \quad\left(v^{*}(0)+x^{*^{\prime}}(0),-x^{*}(0)\right) \in K_{Q_{0}}^{*}(\tilde{x}(0)) \times K_{Q_{1}}^{*}\left(\tilde{x}^{\prime}(0)\right), \\
(d) \quad\left(v^{*}(1)+x^{*^{\prime}}(1),-x^{*}(1)\right) \in \partial_{(x, y)} \varphi\left(\tilde{x}(1), \tilde{x}^{\prime}(1)\right),
\end{gathered}
$$

respectively. Now we are ready to formulate the following optimality theorem.

Theorem 3.1. Suppose that $\varphi$ is a continuous and proper convex function, $F(\cdot, t)$ is a convex set-valued mapping and $Q_{i}(i=0,1)$ are convex sets. Then for optimality of the feasible trajectory $\tilde{x}(t)$ in the problem $\left(P_{C}\right)$ it is sufficient that there exists a pair of absolutely continuous functions $\left\{x^{*}(t), v^{*}(t)\right\}, t \in[0,1]$ satisfying a.e. the second-order Euler-Lagrange type differential inclusion (a)-(b) and the transversality conditions (c), (d) at the initial point $t=0$ and endpoint $t=1$, respectively.

Proof. By the proof idea of Theorem 5.1 [13] from (a), (b) we derive the following inequality

$$
\begin{gathered}
0 \geq\left\langle x^{\prime}(1)-\tilde{x}^{\prime}(1), x^{*}(1)\right\rangle-\left\langle x^{\prime}(0)-\tilde{x}^{\prime}(0), x^{*}(0)\right\rangle \\
-\left\langle v^{*}(1)+x^{*^{\prime}}(1), x(1)-\tilde{x}(1)\right\rangle+\left\langle v^{*}(0)+x^{*^{\prime}}(0), x(0)-\tilde{x}(0)\right\rangle .
\end{gathered}
$$

Now, by definition of dual cones $K_{Q_{0}}^{*}(\tilde{x}(0)), K_{Q_{1}}^{*}\left(\tilde{x}^{\prime}(0)\right)$ from the transversality condition (c) we deduce that

$$
\begin{gathered}
-\left\langle x^{\prime}(0)-\tilde{x}^{\prime}(0), x^{*}(0)\right\rangle+\left\langle v^{*}(0)+x^{*^{\prime}}(0), x(0)-\tilde{x}(0)\right\rangle \geq 0, \\
\forall x(0) \in Q_{0} ; \forall x^{\prime}(0) \in Q_{1} .
\end{gathered}
$$

Thus, it follows from 3.1 and 3.2 that 


$$
0 \geq\left\langle x^{\prime}(1)-\tilde{x}^{\prime}(1), x^{*}(1)\right\rangle-\left\langle v^{*}(1)+x^{*^{\prime}}(1), x(1)-\tilde{x}(1)\right\rangle .
$$

Now, it is not hard to see that the transversality conditions (d) at the endpoint $t=1$, can be rewritten as follows

$$
\begin{gathered}
\varphi\left(x(1), x^{\prime}(1)\right)-\varphi\left(\tilde{x}(1), \tilde{x}^{\prime}(1)\right) \\
\geq\left\langle v^{*}(1)+x^{*^{\prime}}(1), x(1)-\tilde{x}(1)\right\rangle-\left\langle x^{\prime}(1)-\tilde{x}^{\prime}(1), x^{*}(1)\right\rangle .
\end{gathered}
$$

Then, summing the inequalities $3.3,3.4$ for all feasible trajectories $x(\cdot)$ satisfying the initial conditions $x(0) \in Q_{0}, x^{\prime}(0) \in Q_{1}$ we have the needed inequality:

$$
\varphi\left(x(1), x^{\prime}(1)\right)-\varphi\left(\tilde{x}(1), \tilde{x}^{\prime}(1)\right) \geq 0 \text { or } \varphi\left(x(1), x^{\prime}(1)\right) \geq \varphi\left(\tilde{x}(1), \tilde{x}^{\prime}(1)\right) .
$$
$\left(P_{C}^{*}\right)$.

We are now in a position to establish our duality relations between $\left(P_{C}\right)$ and

Remark 3.1. Obviously, in the convex case $H_{F}\left(\cdot, z^{*}\right)$ is concave and the latter definition of LAM coincide with the previous definition. Besides, note that, the LAM (as well as the coderivative of Mordukhovich [19]) is a set-valued extension of the classical notion of the adjoint linear operator in functional analysis.

Remark 3.2. Suppose now we have a problem $\left(P_{C}\right)$ with initial-value problem of Cauchy, where $Q_{0}=\left\{\alpha_{0}\right\}, Q_{1}=\left\{\alpha_{1}\right\}$ and $\alpha_{0}, \alpha_{1}$ are fixed vectors: $\alpha_{i} \in \mathbb{R}^{n}(i=$ $0,1)$. Then the cones of tangent vectors $K_{Q_{0}}(\tilde{x}(0)), K_{Q_{1}}\left(\tilde{x}^{\prime}(0)\right)$ consist of a zero vectors, i.e $K_{Q_{0}}(\tilde{x}(0))=K_{Q_{1}}\left(\tilde{x}^{\prime}(0)\right)=\{0\}$ and the dual to the cones of tangent directions $K_{Q_{0}}^{*}(\tilde{x}(0))=K_{Q_{1}}^{*}\left(\tilde{x}^{\prime}(0)\right)=\mathbb{R}^{n}$. As a consequence, it follows that the condition (c) of theorem is superfluous.

Theorem 3.2. Suppose that the conditions of Theorem 3.1 are satisfied and $\tilde{x}(t)$ is an optimal solution of the primary problem $\left(P_{C}\right)$ with convex DFI. Then a pair of functions $\left\{\tilde{x}^{*}(\cdot), \tilde{v}^{*}(\cdot)\right\}$ is an optimal solution of the dual problem $\left(P_{C}^{*}\right)$ if and only if the conditions $(a)-(d)$ of Theorem 3.1 are satisfied. In addition, the optimal values in the primary $\left(P_{C}\right)$ and dual $\left(P_{C}^{*}\right)$ problems are equal.

Proof. Before all we prove that for all feasible solutions $x(\cdot)$ and dual variables $\left\{x^{*}(\cdot), v^{*}(\cdot)\right\}$ of the primary $\left(P_{C}\right)$ and dual $\left(P_{C}^{*}\right)$ problems, respectively, the inequality holds:

$$
\begin{gathered}
\varphi\left(x(1), x^{\prime}(1)\right) \geq-\varphi^{*}\left(v^{*}(1)+x^{*^{\prime}}(1),-x^{*}(1)\right)+ \\
\int_{0}^{1}\left[M_{F(\cdot, t)}\left(x^{*^{\prime \prime}}(t)+v^{*^{\prime}}(t), v^{*}(t), x^{*}(t)\right)\right] d t \\
-W_{Q_{0}}\left(-v^{*}(0)-x^{*^{\prime}}(0)\right)-W_{Q_{1}}\left(x^{*}(0)\right) .
\end{gathered}
$$

To this end, by using the conjugate $\varphi^{*}$ and definition of Hamiltonian function we can write 


$$
\begin{gathered}
\int_{0}^{1}\left[M_{F(\cdot, t)}\left(x^{*^{\prime \prime}}(t)+v^{*^{\prime}}(t), v^{*}(t), x^{*}(t)\right)\right] d t-W_{Q_{0}}\left(-v^{*}(0)-x^{*^{\prime}}(0)\right) \\
-W_{Q_{1}}\left(x^{*}(0)\right)-\varphi^{*}\left(v^{*}(1)+x^{*^{\prime}}(1),-x^{*}(1)\right) \\
\leq \int_{0}^{1}\left[\left\langle x(t), x^{*^{\prime \prime}}(t)+v^{*^{\prime}}(t)\right\rangle+\left\langle x^{\prime}(t), v^{*}(t)\right\rangle-\left\langle x^{\prime \prime}(t), x^{*}(t)\right\rangle\right] d t \\
+\varphi\left(x(1), x^{\prime}(1)\right)-\left\langle x(1), v^{*}(1)+x^{*^{\prime}}(1)\right\rangle-\left\langle x(1),-x^{*}(1)\right\rangle \\
+\left\langle v^{*}(0)+x^{*^{\prime}}(0), x(0)\right\rangle-\left\langle x^{*}(0), x^{\prime}(0)\right\rangle=\int_{0}^{1}\left[\left\langle x(t), x^{*^{\prime \prime}}(t)\right\rangle-\left\langle x^{\prime \prime}(t), x^{*}(t)\right\rangle\right] d t \\
+\int_{0}^{1} d\left\langle x(t), v^{*}(t)\right\rangle+\varphi\left(x(1), x^{\prime}(1)\right)-\left\langle x(1), v^{*}(1)+x^{*^{\prime}}(1)\right\rangle \\
\quad-\left\langle x^{\prime}(1),-x^{*}(1)\right\rangle+\left\langle v^{*}(0)+x^{*^{\prime}}(0), x(0)\right\rangle-\left\langle x^{*}(0), x^{\prime}(0)\right\rangle \\
=\int_{0}^{1}\left[\left\langle x(t), x^{*^{\prime \prime}}(t)\right\rangle-\left\langle x^{\prime \prime}(t), x^{*}(t)\right\rangle\right] d t+\left\langle x(1), v^{*}(1)\right\rangle-\left\langle x(0), v^{*}(0)\right\rangle \\
+\varphi\left(x(1), x^{\prime}(1)\right)-\left\langle x(1), v^{*}(1)+x^{*^{\prime}}(1)\right\rangle-\left\langle x^{\prime}(1),-x^{*}(1)\right\rangle \\
+\left\langle v^{*}(0)+x^{*^{\prime}}(0), x(0)\right\rangle-\left\langle x^{*}(0), x^{\prime}(0)\right\rangle=\int_{0}^{1}\left[\left\langle x(t), x^{*^{\prime \prime}}(t)\right\rangle-\left\langle x^{\prime \prime}(t), x^{*}(t)\right\rangle\right] d t \\
+\varphi\left(x(1), x^{\prime}(1)\right)-\left\langle x(1), x^{*^{\prime}}(1)\right\rangle-\left\langle x^{\prime}(1),-x^{*}(1)\right\rangle \\
+\left\langle x^{*^{\prime}}(0), x(0)\right\rangle-\left\langle x^{*}(0), x^{\prime}(0)\right\rangle .
\end{gathered}
$$

Further, it is not hard to see that

$$
\begin{gathered}
\int_{0}^{1}\left[\left\langle x^{*^{\prime \prime}}(t), x(t)\right\rangle-\left\langle x^{\prime \prime}(t), x^{*}(t)\right\rangle\right] d t=\int_{0}^{1} d\left[\left\langle x^{*^{\prime}}(t), x(t)\right\rangle-\left\langle x^{\prime}(t), x^{*}(t)\right\rangle\right] \\
=\left\langle x^{*^{\prime}}(1), x(1)\right\rangle-\left\langle x^{\prime}(1), x^{*}(1)\right\rangle-\left\langle x^{*^{\prime}}(0), x(0)\right\rangle+\left\langle x^{\prime}(0), x^{*}(0)\right\rangle .
\end{gathered}
$$

Then the relationships (3.6) and (3.7) give us

$$
\begin{gathered}
\int_{0}^{1}\left[M_{F(\cdot, t)}\left(x^{*^{\prime \prime}}(t)+v^{*^{\prime}}(t), v^{*}(t), x^{*}(t)\right)\right] d t-W_{Q_{0}}\left(-v^{*}(0)-x^{*^{\prime}}(0)\right) \\
-W_{Q_{1}}\left(x^{*}(0)\right)-\varphi^{*}\left(v^{*}(1)+x^{*^{\prime}}(1),-x^{*}(1)\right) \leq\left\langle x^{*^{\prime}}(1), x(1)\right\rangle-\left\langle x^{\prime}(1), x^{*}(1)\right\rangle \\
-\left\langle x^{*^{\prime}}(0), x(0)\right\rangle+\left\langle x^{\prime}(0), x^{*}(0)\right\rangle+\varphi\left(x(1), x^{\prime}(1)\right)-\left\langle x(1), x^{*^{\prime}}(1)\right\rangle \\
-\left\langle x^{\prime}(1),-x^{*}(1)\right\rangle+\left\langle x^{*^{\prime}}(0), x(0)\right\rangle-\left\langle x^{*}(0), x^{\prime}(0)\right\rangle=\varphi\left(x(1), x^{\prime}(1)\right)
\end{gathered}
$$

and this proves the inequality (3.5). Furthermore, suppose that a pair $\left\{\tilde{x}^{*}(\cdot), \tilde{v}^{*}(\cdot)\right\}$ satisfies the conditions (a)-(d) of Theorem 3.1. Then by definition of LAM the Euler-Lagrange type inclusion (a) and the condition (b) imply that

$$
\begin{gathered}
H_{F}\left(x(t), x^{\prime}(t), x^{*}(t)\right)-H_{F}\left(\tilde{x}(t), \tilde{x}^{\prime}(t), \tilde{x}^{*}(t)\right) \\
\leq\left\langle\tilde{x}^{*^{\prime \prime}}(t)+\tilde{v}^{*^{\prime}}(t), x(t)-\tilde{x}(t)\right\rangle+\left\langle\tilde{v}^{*}(t), x^{\prime}(t)-\tilde{x}^{\prime}(t)\right\rangle,
\end{gathered}
$$

whence by the definition of function $M_{F}$ we deduce that

$$
\begin{gathered}
\left\langle\tilde{x}^{*^{\prime \prime}}(t)+\tilde{v}^{*^{\prime}}(t), \tilde{x}(t)\right\rangle+\left\langle\tilde{v}^{*}(t), \tilde{x}^{\prime}(t)\right\rangle-H_{F}\left(\tilde{x}(t), \tilde{x}^{\prime}(t), \tilde{x}^{*}(t)\right) \\
=M_{F}\left(\tilde{x}^{*^{\prime \prime}}(t)+\tilde{v}^{*^{\prime}}(t), \tilde{v}^{*}(t), \tilde{x}^{*}(t)\right) .
\end{gathered}
$$

On the other hand, by the transversality condition (c) we can write 


$$
\begin{gathered}
-\left\langle\tilde{v}^{*}(0)+\tilde{x}^{*^{\prime}}(0), \tilde{x}(0)\right\rangle=W_{Q_{0}}\left(-\tilde{v}^{*}(0)-\tilde{x}^{*^{\prime}}(0)\right), \\
\left\langle\tilde{x}^{*}(0), \tilde{x}^{\prime}(0)\right\rangle=W_{Q_{1}}\left(\tilde{x}^{*}(0)\right)
\end{gathered}
$$

Finally, by Theorem 1.27 [8] the transversality condition (d) is equivalent to the relation

$$
\begin{aligned}
\varphi^{*}\left(\tilde{v}^{*}(1)\right. & \left.+\tilde{x}^{*^{\prime}}(1),-\tilde{x}^{*}(1)\right)=\left\langle\tilde{x}(1), \tilde{v}^{*}(1)+\tilde{x}^{*^{\prime}}(1)\right\rangle \\
& +\left\langle\tilde{x}^{\prime}(1), \tilde{x}^{*}(1)\right\rangle-\varphi\left(\tilde{x}(1), \tilde{x}^{\prime}(1)\right) .
\end{aligned}
$$

Thus, taking into account the relationships (3.8)-(3.10) in (3.6) the inequality sign is replaced by equality and for $\tilde{x}(\cdot)$ and $\left\{\tilde{x}^{*}(\cdot), \tilde{v}^{*}(\cdot)\right\}$ the equality of values of the primary and dual problems is ensured. Moreover, $\tilde{x}(\cdot)$ and $\left\{\tilde{x}^{*}(\cdot), \tilde{v}^{*}(\cdot)\right\}$ are satisfies the conditions (a)-(d) of Theorem 3.1 and the collection (a)-(d) is a dual relation for the primary $\left(P_{C}\right)$ and dual $\left(P_{C}^{*}\right)$ problems.

Remark 3.3. It is interesting to note that in the theory of mathematical programming problem the analogy of these results consists of the following. Suppose that we have a problem

$$
\inf _{x \in A} f(x)
$$

where $f$ is a closed, proper convex function and that $A$ is a convex closed set. It is known from convex analysis that the operations of addition and infimal convolution of convex functions are dual to each other [7, 8]. By this result, if there exists a point $u^{0} \in A$, where $f$ is continuous ( $f$ is continuous on ridom $f$, however, $f$ may have a point of discontinuity in its boundary), the optimal value of problem $(\mathrm{G})$ is

$$
\begin{gathered}
\inf _{u \in A} f(u)=\inf \left\{f(u)+\delta_{A}(u)\right\}=-\sup \left\{-f(u)-\delta_{A}(u)\right\} \\
=-\sup \left\{\langle u, 0\rangle-\left[f(u)+\delta_{A}(u)\right]\right\}=-\left(f+\delta_{A}\right)^{*}(0)=-\left(f^{*} \oplus \delta_{A}^{*}\right)(0) \\
=-\inf \left\{f^{*}\left(u^{*}\right)+\delta_{A}^{*}\left(-u^{*}\right)\right\}=\sup \left\{-f^{*}\left(u^{*}\right)-\delta_{A}^{*}\left(-u^{*}\right)\right\},
\end{gathered}
$$

where $\delta_{A}(\cdot)$ is the indicator function of $A$.

In general, it can be noticed that $\left(f+\delta_{A}\right)^{*}(0) \leq\left(f^{*} \oplus \delta_{A}^{*}\right)(0)$ and so

$$
\inf _{u \in A} f(u) \geq \sup \left\{-f^{*}\left(u^{*}\right)-\delta_{A}^{*}\left(-u^{*}\right)\right\} .
$$

Then it is reasonable to announce that the dual problem to the primary problem (3.4) has the form

$$
\sup \left\{-f^{*}\left(u^{*}\right)-\delta_{A}^{*}\left(-u^{*}\right)\right\} . \quad\left(G^{*}\right)
$$

In addition, if the value of the problem $(\mathrm{G})$ is finite, then the supremum in the problem $\left(G^{*}\right)$ is attained for all $u^{*}$. The problem $\sup \left\{-f^{*}\left(u^{*}\right)-\delta_{A}^{*}\left(-u^{*}\right)\right\}\left(G^{*}\right)$ we call the dual problem to the primary problem $(\mathrm{G})$. Then if a pair $\left(x, x^{*}\right)$ of points $x$ and $x^{*}$ is such that $x^{*} \in \partial F(x) \cap K_{A}^{*}(x)$, then $x$ and $x^{*}$ are solutions of the problems $(\mathrm{G})$ and $\left(G^{*}\right)$, respectively (see, for example $[7,8]$ ). Consequently, the results of Sections 2,3 have an important theoretical significance. In practise, we should solve only the dual problems $\sup \left(P_{C}^{*}\right)$ similar the linear dual programming problem. 
Example 3.1. (Second-order linear optimal control problems). Suppose we have the linear continuous case of second-order DFI:

$$
\begin{gathered}
\text { infimum } \varphi\left(x(1), x^{\prime}(1)\right), \\
\text { subject to } \quad x^{\prime \prime}(t)=A_{0} x(t)+A_{1} x^{\prime}(t)+B u(t), u(\cdot) \in U, \\
x(0) \in Q_{0}, x^{\prime}(0) \in Q_{1}, t \in[0,1],
\end{gathered}
$$

where $A_{0}, A_{1}$ and $B$ are $n \times n$ and $n \times r$ matrices, respectively, the function $\varphi$ and $Q_{i}(i=0,1)$ are the same, $U \subseteq \mathbb{R}^{r}$ is a convex compact. The problem is to find a controlling parameter $\tilde{u}(t) \in U$ such that the arc $\tilde{x}(t)$ corresponding to it minimizes $\varphi\left(x(1), x^{\prime}(1)\right)$. We introduce a set-valued mapping of the form $F(x, y)=A_{0} x+A_{1} y+B U$. Then it is easy to see that

$$
\begin{gathered}
M_{F}\left(x^{*}, y^{*}, z^{*}\right)=\inf _{(x, y, z) \in \operatorname{gph} F}\left\{\left\langle x, x^{*}\right\rangle+\left\langle y, y^{*}\right\rangle-\left\langle z, z^{*}\right\rangle\right\} \\
=\inf _{x, y}\left[\left\langle x, x^{*}-A_{0}^{*} z^{*}\right\rangle+\left\langle y, y^{*}-A_{1}^{*} z^{*}\right\rangle\right]-\sup _{u \in U}\left\langle u, B^{*} z^{*}\right\rangle \\
= \begin{cases}-W_{U}\left(B^{*} z^{*}\right), & \text { if } x^{*}=A_{0}^{*} z^{*}, y^{*}=A_{1}^{*} z^{*}, \\
-\infty, & \text { otherwise. }\end{cases}
\end{gathered}
$$

Then using the formula for $M_{F}$, in view of the dual problem $\left(P_{C}^{*}\right)$ we can write

$$
\begin{gathered}
M_{F}\left(x^{*^{\prime \prime}}(t)+v^{*^{\prime}}(t), v^{*}(t), x^{*}(t)\right) \\
= \begin{cases}-W_{U}\left(B^{*} x^{*}(t)\right), & \text { if } x^{*^{\prime \prime}}(t)+v^{*^{\prime}}(t)=A_{0}^{*} x^{*}(t), v^{*}(t)=A_{1}^{*} x^{*}(t), \\
-\infty, & \text { otherwise. }\end{cases}
\end{gathered}
$$

or, more compactly,

$$
\begin{gathered}
M_{F}\left(x^{*^{\prime \prime}}(t)+v^{*^{\prime}}(t), v^{*}(t), x^{*}(t)\right) \\
= \begin{cases}-W_{U}\left(B^{*} x^{*}(t)\right), & \text { if } x^{*^{\prime \prime}}(t)=A_{0}^{*} x^{*}(t)-A_{1}^{*} x^{*^{\prime}}(t), \\
-\infty, & \text { otherwise. }\end{cases}
\end{gathered}
$$

Then the dual problem of problem (3.11) is

$$
\begin{aligned}
\sup _{x^{*}(\cdot)}\{ & -\varphi^{*}\left(A_{1}^{*} x^{*}(1)+x^{*^{\prime}}(1),-x^{*}(1)\right)-\int_{0}^{1} W_{U}\left(B^{*} x^{*}(t)\right) d t \\
& \left.-W_{Q_{0}}\left(-A_{1}^{*} x^{*}(0)-x^{*^{\prime}}(0)\right)-W_{Q_{1}}\left(x^{*}(0)\right)\right\},
\end{aligned}
$$

where $x^{*}(\cdot)$ is a solution of the adjoint Euler-Lagrange inclusion/equation

$$
x^{*^{\prime \prime}}(t)=A_{0}^{*} x^{*}(t)-A_{1}^{*} x^{*^{\prime}}(t)
$$

Consequently, maximization in this dual problem to primary problem (3.11) is realized over the set of solutions of the adjoint equation. 


\section{Duality in Problems with Second-Order Polyhedral DFIs}

In this section we establish the dual problem $\left(P L^{*}\right)$ to the problem with the following second-order polyhedral differential inclusion:

$$
\begin{aligned}
& \text { infimum } \varphi\left(x(1), x^{\prime}(1)\right), \\
x^{\prime \prime}(t) & \in F\left(x(t), x^{\prime}(t), t\right), \text { a.e. } t \in[0,1], \\
x(0) \in Q_{0}, x^{\prime}(0) & \in Q_{1}, F(x, y, t)=\{z: A x+B y-C z \leq d\},
\end{aligned}
$$

where $F$ is a second-order polyhedral set-valued mapping, $A, B$ and $C$ are $s \times n$ dimensional matrices, $d$ is a $s$-dimensional column-vector, $\varphi: \mathbb{R}^{2 n} \rightarrow \mathbb{R}^{1}$ is a convex function, $Q_{0}, Q_{1}$ are nonempty convex subsets of $\mathbb{R}^{n}$. We label this problem by $(P L)$. Then with respect to the dual problem $\left(P_{C}^{*}\right)$ first we should calculate $M_{F}\left(x^{*}, y^{*}, z^{*}\right)$ :

$$
M_{F}\left(x^{*}, y^{*}, z^{*}\right)=\inf \left\{\left\langle x, x^{*}\right\rangle+\left\langle y, y^{*}\right\rangle-\left\langle z, z^{*}\right\rangle:(x, y, z) \in g p h F\right\} .
$$

In fact, denoting $w=(x, y, z) \in \mathbb{R}^{3 n}, w^{*}=\left(x^{*}, y^{*},-z^{*}\right) \in \mathbb{R}^{3 n}$ we have a linear programming problem

$$
\inf \left\{\left\langle w, w^{*}\right\rangle: D w \leq d\right\}
$$

where $D=[A: B:-C]$ is $s \times 3 n$ block matrix. Then according to the linear programming theory if $\tilde{w}=(\tilde{x}, \tilde{y}, \tilde{z})$ is a solution of $(4.2)$, then there exists $s$ dimensional vector $\lambda \geq 0$ such that

$$
w^{*}=-D^{*} \lambda,\langle A \tilde{x}+B \tilde{y}-C \tilde{z}-d, \lambda\rangle=0 .
$$

On the contrary, if these conditions are satisfied, then $\tilde{w}=(\tilde{x}, \tilde{y}, \tilde{z})$ is a solution of the problem (4.2).

Hence, $w^{*}=-D^{*} \lambda$ means that $x^{*}=-A^{*} \lambda, y^{*}=-B^{*} \lambda, z^{*}=-C^{*} \lambda, \lambda \geq 0$. Thus, we find that

$$
\begin{gathered}
M_{F}\left(x^{*}, y^{*}, z^{*}\right)=\left\langle\tilde{x},-A^{*} \lambda\right\rangle+\left\langle\tilde{y},-B^{*} \lambda\right\rangle-\left\langle\tilde{z},-C^{*} \lambda\right\rangle \\
=-\langle A \tilde{x}, \lambda\rangle-\langle B \tilde{y}, \lambda\rangle+\langle C \tilde{z}, \lambda\rangle=-\langle d, \lambda\rangle .
\end{gathered}
$$

On the other hand, from the form of $M_{F}\left(x^{*^{\prime \prime}}(t)+v^{*^{\prime}}(t), v^{*}(t), x^{*}(t)\right)$ by Theorem 3.1 we derive that

$$
x^{*^{\prime \prime}}(t)+v^{*^{\prime}}(t)=-A^{*} \lambda(t), v^{*}(t)=-B^{*} \lambda(t), x^{*}(t)=-C^{*} \lambda(t), \lambda(t) \geq 0
$$

or

$$
C^{*} \lambda^{\prime \prime}(t)+B^{*} \lambda^{\prime}(t)-A^{*} \lambda(t)=0, \lambda(t) \geq 0 .
$$

Therefore, taking into account (4.3)-(4.5) and Theorem 3.2 we have the following dual problem: 


$$
\begin{aligned}
\sup _{\lambda(t) \geq 0} & \left\{-\varphi^{*}\left(-C^{*} \lambda(1)-B^{*} \lambda(1), C^{*} \lambda(1)\right)-\int_{0}^{1}\langle d, \lambda(t)\rangle d t\right. \\
- & \left.W_{Q_{0}}\left(C^{*} \lambda^{\prime}(0)+B^{*} \lambda(0)\right)-W_{Q_{1}}\left(-C^{*} \lambda(0)\right)\right\} .
\end{aligned}
$$

Now, before formulation of duality theorem we should proof sufficient condition of optimality for a problem $(P L)$.

Theorem 4.1. Let $\varphi: \mathbb{R}^{2 n} \rightarrow \mathbb{R}^{1}$ be continuous proper convex function and $F$ be a polyhedral set-valued mapping given in problem $(P L)$. Moreover, let $Q_{0}, Q_{1}$ be convex sets. Then for the optimality of the trajectory $\tilde{x}(\cdot)$ in problem $(P L)$ with second-order polyhedral differential inclusions, it is sufficient that there exists a function $\lambda(t) \geq 0, t \in[0,1]$ satisfying a.e. the following second-order EulerLagrange type polyhedral differential inclusion and transversality conditions at the endpoints $t=0$ and $t=1$ :

$$
\begin{aligned}
& C^{*} \lambda^{\prime \prime}(t)+B^{*} \lambda^{\prime}(t)-A^{*} \lambda(t)=0, \lambda(t) \geq 0, \\
& \left\langle A \tilde{x}(t)+B \tilde{x}^{\prime}(t)-C \tilde{x}^{\prime \prime}(t)-d, \lambda(t)\right\rangle=0, \text { a.e. } t \in[0,1] \\
& -C^{*} \lambda^{\prime}(0)-B^{*} \lambda(0) \in K_{Q_{0}}^{*}(\tilde{x}(0)) ; C^{*} \lambda(0) \in K_{Q_{1}}^{*}\left(\tilde{x}^{\prime}(0)\right), t=0, \\
& \left(-C^{*} \lambda^{\prime}(1)-B^{*} \lambda(1), C^{*} \lambda(1)\right) \in \partial \varphi\left(\tilde{x}(1), \tilde{x}^{\prime}(1)\right), t=1 .
\end{aligned}
$$

Proof. In fact, condition (2.1) of the theorem is formula (4.5). Further, by the conditions (c), (d) of Theorem 3.1 we have

$$
\begin{gathered}
x^{*^{\prime}}(0)+v^{*}(0) \in K_{Q_{0}}^{*}(\tilde{x}(0)) ;-x^{*}(0) \in K_{Q_{1}}^{*}\left(\tilde{x}^{\prime}(0)\right), \\
\left(x^{*^{\prime}}(1)+v^{*}(1),-x^{*}(1)\right) \in \partial \varphi\left(\tilde{x}(1), \tilde{x}^{\prime}(1)\right) .
\end{gathered}
$$

Since by (4.4) $v^{*}(t)=-B^{*} \lambda(t), x^{*}(t)=-C^{*} \lambda(t)$, we derive from (4.6), (4.7) that

$$
\begin{gathered}
-C^{*} \lambda^{\prime}(0)-B^{*} \lambda(0) \in K_{Q_{0}}^{*}(\tilde{x}(0)) ; C^{*} \lambda(0) \in K_{Q_{1}}^{*}\left(\tilde{x}^{\prime}(0)\right), \\
\left(-C^{*} \lambda^{\prime}(1)-B^{*} \lambda(1), C^{*} \lambda(1)\right) \in \partial \varphi\left(\tilde{x}(1), \tilde{x}^{\prime}(1)\right) .
\end{gathered}
$$

The proof of theorem is completed.

Then, as a result of Theorems 3.1 and 3.2 for a problem $(P L)$ we have the following duality theorem.

Theorem 4.2. Let the conditions of Theorem 3.1 be satisfied and $\tilde{x}(\cdot)$ be an optimal solution of the primary problem $(P L)$. Then $\tilde{x}^{*}(t), t \in[0,1]$ is an optimal solution of the dual problem $\left(P L^{*}\right)$ if and only if the sufficient optimality conditions of Theorem 3.1 are satisfied. In addition, the optimal values in the primary $(P L)$ and dual $\left(P L^{*}\right)$ problems are equal. 


\section{Conclusion}

First are derived necessary and sufficient optimality conditions in the form of Euler-Lagrange type inclusions and transversality conditions. Then we treat dual results according to the dual operations of addition and infimal convolution of convex functions. For construction of the duality problem skilfully computation of conjugate and support functions are required. It appears that the Euler-Lagrange type inclusions are duality relations for both primary and dual problems and that the dual problem for discrete-approximate problem make a bridge between the dual problems of discrete and continuous problems. We believe that relying to the method described in this paper it can be obtained the similar duality results to optimal control problems with any higher order differential inclusions. In this way for computation of the conjugate function and support function of discrete-approximate problem a Pascal triangle with binomial coefficients, can be successfully used for any "higher order" calculations. These difficulties, of course, are connected with the existence of higher order difference derivatives in Mayer functional and discrete-approximate inclusions, respectively. There has been a significant development in the study of duality theory to problems with first order differential/difference inclusions in recent years. As an open problem for further investigations, we mention the study of duality theory for an arbitrary higher-order differential inclusion. Besides, there can be no doubt that investigations of duality results to problems with second-order differential inclusions can have great contribution to the modern development of the optimal control theory. Consequently, there arises a rather complicated problem with simultaneous determination of conjugacy of a Mayer functional depending of high order derivatives of searched functions. Thus, we can conclude that the proposed method is reliable for solving the various duality problems with higher order discrete and differential inclusions.

\section{References}

[1] A.Auslender, J.Mechler, Second-order viability problems for differential inclusions, J.Math.Anal.Appl., 181 (1994), 205-218.

[2] Artstein-Avidan, V.Milman, A characterization of the concept of duality, Elect. Research Announc., 14 (2007), 42-59.

[3] A.Dhara, A.Mehra, Conjugate duality for generalized convex optimization problems, J. Indust. Manag. Optim., 3 (2007), 415-427.

[4] O.Dovgoshey, O.Martio, V.Ryazanov, M.Vuorinen, The Cantor function, Expo. Math., 24 (2006), 1-37.

[5] N.C.Kourogenis, Strongly nonlinear second-order differential inclusions with generalized boundary conditions, J. Math. Anal. Appl., 287 (2003), 348-364.

[6] S.Kyritsi, N.Matzakos, N.S.Papageorgiou, Periodic problems for strongly nonlinear second-order differential inclusions, J. Diff. Eq., 183 (2002), 279-302.

[7] P.J.Laurent, Approximation et optimisation, Herman, Paris, 1972.

[8] E.N.Mahmudov, Approximation and Optimization of Discrete and Differential Inclusions, Elsevier, Boston, USA, 2011.

[9] E.N.Mahmudov, On duality in problems of optimal control described by convex differential inclusions of Goursat-Darboux type, J. Math. Anal. Appl., 307 (2005), 628-640. 
[10] E.N.Mahmudov, M.E.Unal, Optimal control of discrete and differential inclusions with distributed parameters in the gradient form, J. Dynam. Contr. Syst., 18 (2012), 83-101

[11] E.N.Mahmudov, Convex optimization of second-order discrete and differential inclusions with inequality constraints, J. Convex Anal., 25 (2018), 1-26.

[12] E.N.Mahmudov, Optimization of Fourth-Order Differential Inclusions, Proceed. Inst. Math. Mech. ANAS, 44 (2018), 90-106.

[13] E.N.Mahmudov, Optimization of Mayer problem with Sturm-Liouville type differential inclusions, J. Optim. Theory Appl. (JOTA), 177 (2018), 345-375.

[14] E.N.Mahmudov, Optimization of fourth order Sturm-Liouville type differential inclusions with initial point constraints, J. Industr. Manag. Optim. (JIMO), (2018),1335

[15] E.N.Mahmudov, Optimal control of second-order delay-discrete and delaydifferential inclusions with state constraints, Evol. Equ. Contr. Theory (EECT), 7 (2018), 501-529.

[16] E.N.Mahmudov, Optimal control of higher order differential inclusions with functional constraints, ESAIM: COCV, doi: https://doi.org/10.1051/cocv/2019018 (Institute of Henri Poincare).

[17] M.J.Mardanov, T.K.Melikov, A Method for Studying the Optimality of Controls in Discrete Systems, Proceed. Inst. Math. Mech. ANAS, 40 (2014), 5-13.

[18] M.J.Mardanov, M.T.Samin, N.I.Mahmudov, On the theory of necessary optimality conditions in discrete systems, Adv. Differ. Equat., 2015 (2015), 1-15.

[19] B.S.Mordukhovich, Variational Analysis and Generalized Differentiation, I: Basic Theory; II: Applications, Grundlehren Series (Fundamental Principles of Mathematical Sciences), Vol. 330 and 331, Springer-Verlag Berlin Heidelberg, 2006.

[20] R.T.Rockafellar, P.R.Wolenski, Convexity in Hamilton-Jacobi theory 1: Dynamics and duality, SIAM J. Contr. Optim., 39 (2000), 1323-1350.

[21] Tan H. Cao, B.S.Mordukhovich, Optimal control of a nonconvex perturbed sweeping process, J. Differ. Equ., 266 (2019),1003-1050.

[22] Q.Zhang, G.Li, Nonlinear boundary value problems for second-order differential inclusions, Nonlin. Anal.: Theory, Methods Appl., 70 (2009), 3390-3406.

Elimhan N. Mahmudov

Department of Mathematics, Istanbul Technical University, 34469 Maslak, Istanbul, Turkey

Institute of Control Systems, Azerbaijan National Academy of Sciences, AZ1141, Baku, Azerbaijan

E-mail address: elimhan22@yahoo.com

Misir J. Mardanov

Institute of Mathematics and Mechanics, Azerbaijan National Academy of Sciences, AZ1141, Baku, Azerbaijan

E-mail address: misirmardanov@yahoo.com

Received: November 13, 2019; Revised: March 2, 2020; Accepted: March 7, 2020 